This item was submitted to Loughborough's Research Repository by the author.

Items in Figshare are protected by copyright, with all rights reserved, unless otherwise indicated.

\title{
A live system for wavelet compression of high speed computer network measurements
}

\section{PLEASE CITE THE PUBLISHED VERSION}

PUBLISHER

(C) Springer-Verlag

LICENCE

CC BY-NC-ND 4.0

\section{REPOSITORY RECORD}

Kyriakopoulos, Konstantinos G., and David J. Parish. 2019. "A Live System for Wavelet Compression of High Speed Computer Network Measurements". figshare. https://hdl.handle.net/2134/3019. 
This item was submitted to Loughborough's Institutional Repository by the author and is made available under the following Creative Commons Licence conditions.

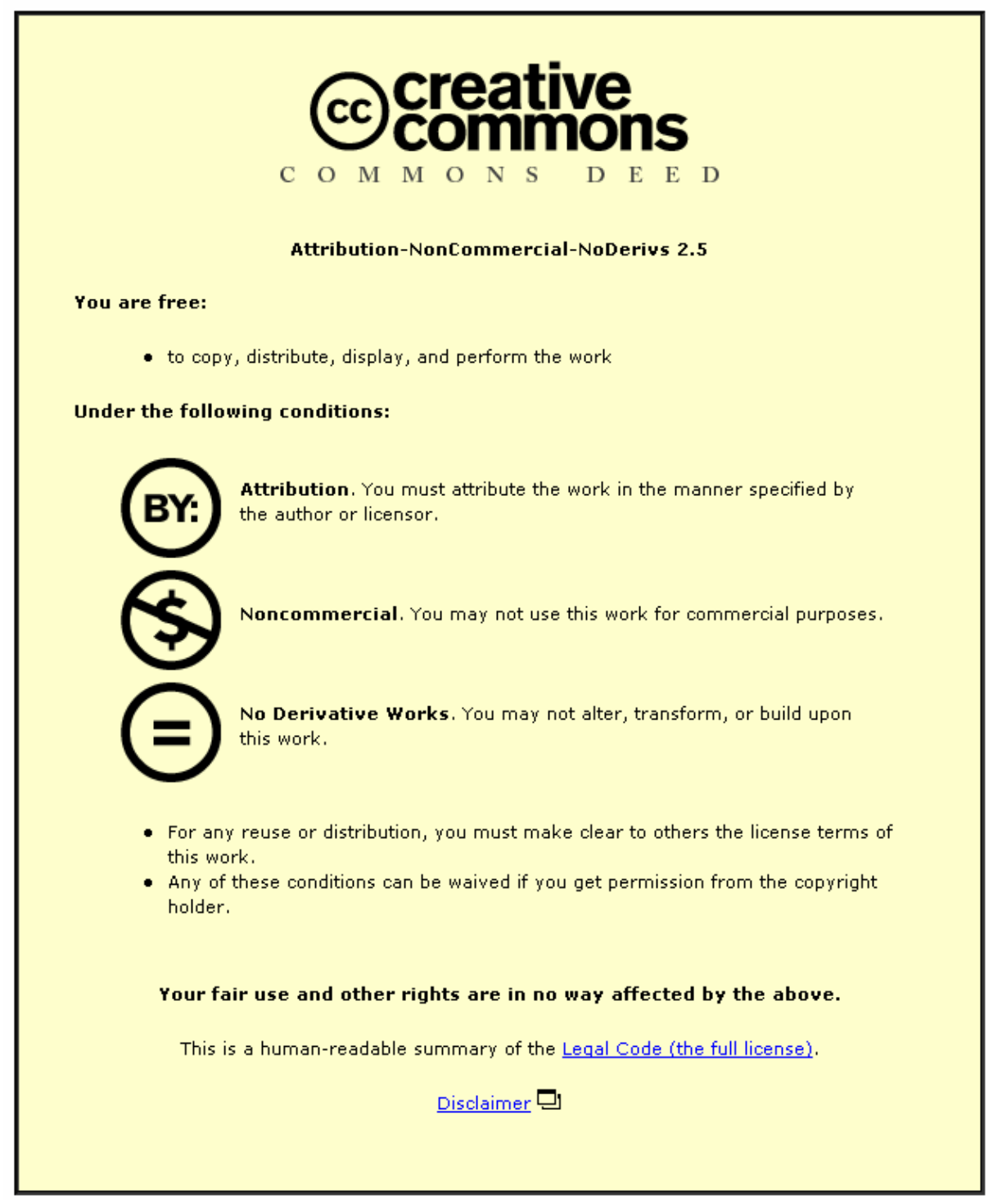

For the full text of this licence, please go to: http://creativecommons.org/licenses/by-nc-nd/2.5/ 


\title{
A Live System for Wavelet Compression of High Speed Computer Network Measurements
}

\author{
Konstantinos Kyriakopoulos, David J. Parish \\ High Speed Networks, Electronic and Electrical Engineering, \\ Loughborough University, Loughborough, Leicestershire LE11 3TU, U.K. \\ $\{k . k y r i a k o p o u l o s, d . j \cdot p a r i s h\} @ l b o r o . a c . u k$ \\ http://www-staff.lboro.ac.uk/ elkk/
}

\section{Introduction}

Monitoring high-speed networks for a long period of time produces a high volume of data, making the storage of this information practically inefficient. To this end, there is a need to derive an efficient method of data analysis and reduction in order to archive and store the enormous amount of monitored traffic.

Satisfying this need is useful not only for administrators but also for researchers who run their experiments on the monitored network. The researchers would like to know how their experiments affect the network's behavior in terms of utilization, delay, packet loss, data rate etc.

In this paper a method of compressing computer network measurements while preserving the quality in interesting signal characteristics is presented. Eight different mother wavelets are compared against each other in order to examine which one offers the best results in terms of quality in the reconstructed signal. The proposed wavelet compression algorithm is compared against the lossless compression tool bzip2 in terms of compression ratio (C.R.). Finally, practical results are presented by compressing sampled traffic recorded from a live network.

\section{Methodology}

Wavelet analysis is not a compression tool but a transformation to a domain that provides a different view of the data that is more eligible to compression than the original data itself. This happens because small wavelet coefficients can be discarded without a significant loss in the quality of the signal. On the other hand, large coefficients represent important characteristics of the signal and they should be kept.

Gupta and Kaur [1] proposed an adaptive thresholding technique that is calculated from the value of the wavelet coefficients. This scheme is not based on signal denoising but rather tries to statistically identify significant coefficients.

Afterwards, normalization and run length encoding are applied. For the simulation experiments thirty delay and thirty data rate signals of 1024 points were used. The delay signals were measured over the test bed of High Speed Networks (HSN) research group. The data rate signals are from a real commercial network. 


\section{Wavelet Comparison}

Eight wavelets were chosen and compared against each other in order to find out which one offers better reconstruction results. The following wavelets were compared: Haar, Meyer, Biorthogonal 3.9 and Daubechies D4, D6, D8, D10, D12. The index of Daubechies wavelets indicates the number of coefficients. The number of vanishing moments each Daubechies wavelet has is half of the number of coefficients.

Wavelets with many vanishing moments are described with many coefficients in the scaling and wavelet functions, thus increasing the computation overhead of the wavelet transform, the complexity of the algorithm and the output file size. Table 1 shows the average PSNR value after reconstruction at level 6 for thirty delay and data rate signals.

Table 1. Average PSNR for delay and data rate signals after reconstruction at level 6

\begin{tabular}{|c|c|c|c|c|c|c|c|c|}
\hline Wavelet & Haar & D4 & D6 & D8 & D10 & D12 & Meyer & Bio3.9 \\
\hline $\begin{array}{c}\text { PSNR (db) } \\
\text { Delay }\end{array}$ & 39.60 & 38.25 & 37.65 & 37.47 & 37.05 & 36.97 & 37.08 & 37.35 \\
\hline $\begin{array}{c}\text { PSNR (db) } \\
\text { Data Rate }\end{array}$ & 55.16 & 54.06 & 53.99 & 50.69 & 52.59 & 53.02 & 54.91 & 51.72 \\
\hline
\end{tabular}

The Haar wavelet provides higher PSNR values for the reconstructed signals in both delay and data rate signals and has the following advantages: It is conceptually simple, fast, memory efficient and exactly reversible without producing edge effects.

\section{Simulation Results}

Fig. 1a shows a delay signal, before and after the compression. Because the two signals are very similar, the error between them is also provided for better judgment (lower line). The signal is decomposed at level 10 and the reconstruction quality is $37.85 \mathrm{~dB}$ while the C.R. is 13.7. PSNR values less than $35 \mathrm{~dB}$ loose some of the important signal characteristics while PSNR values less than $30 \mathrm{~dB}$ are not acceptable for such signals.

Fig. 1b shows a more interesting case of a data rate signal. This signal includes a spike, which is kept intact after the compression. A characteristic of the proposed algorithm is that it detects the spike as a more interesting feature than the rest of the signal. As a result, the algorithm's first priority becomes to preserve this characteristic and then comes the rest of the signal. The PSNR is $35 \mathrm{~dB}$ and the C.R.=26.57.

Fig. 2 compares the C.R. results of the suggested wavelet technique against bzip2. It is interesting to examine the results from wavelet transform against a non-transform compression technique. In average, for delay signals (Fig. 2a) the suggested method (WT) achieves compression 6.5 times more than bzip2 with the best score being 11 times and the worst score 2.3 times. For data rate signals (Fig. 2b) the average compression is 4.7 times more than bzip2 with the best score being 12 times and the worst 4 times. 


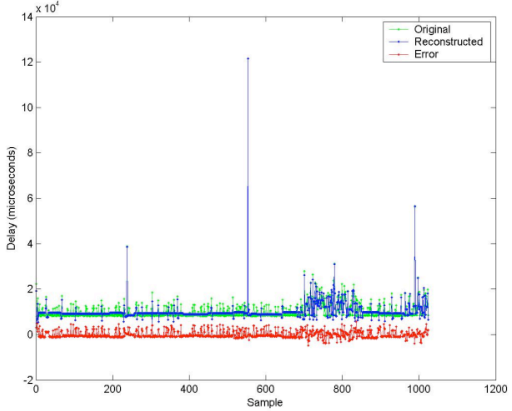

(a)

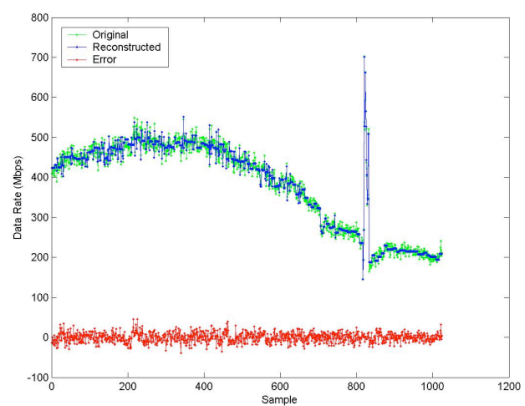

(b)

Fig. 1. (a) Delay signal 30 decomposed at level 10, PSNR $=37.85$ dB. (b) Data rate signal 16 analyzed at level 5 with PSNR $=35.4 \mathrm{~dB}$

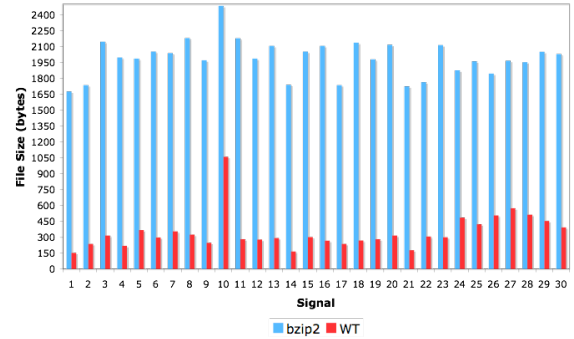

(a)

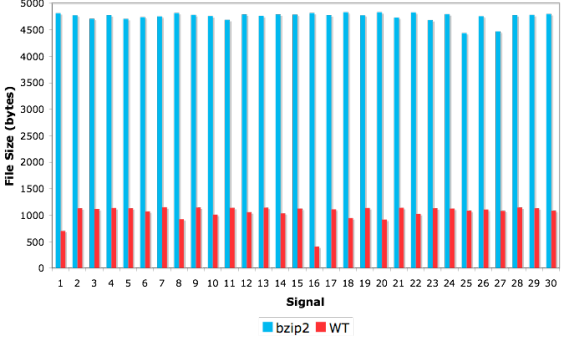

(b)

Fig. 2. Compression performance of the wavelet algorithm against bzip2 for delay (a) and data rate (b) signals. Each examined signal is located on the $\mathrm{x}$-axis. The $\mathrm{y}$-axis shows the file size in bytes.

\section{Practical Results}

The full algorithm is already implemented in CoMo. CoMo is a passive monitoring platform developed for the purpose of monitoring network links at high speeds and replying to real-time queries regarding network statistics. CoMo has various modules that each calculates one or more network measurements [2]. The proposed algorithm can be imbedded in the modules and compress these measurements. When CoMo receives a query, the information is first decompressed and then shown to the end user.

The experiment lasted for 8 days and CoMo was monitoring traffic recorded at HSN research group's live network. The overall achieved compression is 34.5 times. Fig. 3 presents a segment of 34 minutes from the 8 days duration experiment. This signal is characterized by discrete bursts of data rate. Some have amplitude of $70 \mathrm{kB} / \mathrm{s}$ while others are half that size or less. The reconstruction keeps intact the peaks and 
smoothes out the relatively small variation of the signal. PSNR for that segment is $55.9 \mathrm{~dB}$.

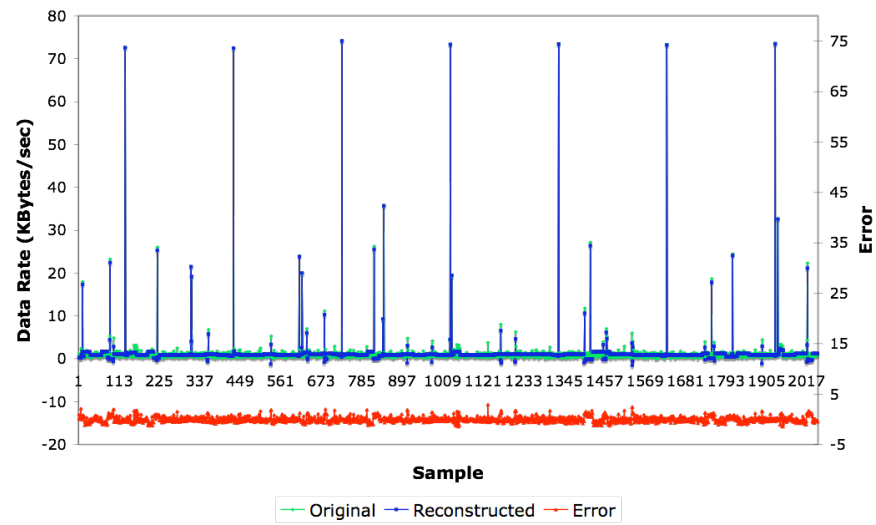

Fig. 3. Segment of 34 minutes of a data rate signal compressed live by CoMo. Error is given on the secondary $y$-axis on the right.

\section{Conclusions - Future Work}

This paper proposes the use of wavelet analysis techniques along with a wavelet coefficient thresholding method for compressing computer network measurements such as data rate and delay. Even though the compression is lossy, the important characteristics of the examined signal are preserved. In order to increase the compression, the detail characteristics are smoothed out by discarding the corresponding detail coefficients.

An evaluation of various wavelets with increasing vanishing moments was presented in order to determine which wavelet is more appropriate for performing the analysis. From simulation results, the Haar wavelet is found to be the best option as it offers the best results in terms of quality and compression ratio

However, some improvements should be done in how the algorithm deals with the threshold in cases that spikes occur in an already bursty signal like in signal 16 (Fig. 1b). This would lead to more control over the quality of the reconstructed signal and more consistent PSNR values.

\section{References}

1. Savita Gupta and Lakhwinder Kaur, "Wavelet Based Image Compression using Daubechies Filters", In proc. 8th National conference on communications, I.I.T. Bombay, NCC-2002

2. Gianluca Iannaccone, Christopher Diot, Derek McAulley, Andrew Moore, Ian Pratt, Luigi Rizzo, " The CoMo White Paper", INTEL research technical report 